\title{
Examining Key Notions for Method Adaptation
}

\author{
Mehmet N. Aydin \\ University of Twente, Department of Information Systems and Change \\ Management. P. O. Box 217, 7500 AE, Enschede, The Netherlands, \\ m.n.aydin@utwente.nl
}

\begin{abstract}
It is a well-known fact that IS development methods are not used as prescribed in actual development projects. That is, every ISD method in a development project is subject to its modifications because its peculiarities and emerging situations cannot be understood adequately in a prescribed manner. Though the idea of method modifications has been studied exclusively under the subject matter called situational method development, the underlying notions (situation, context, agency, and method fragment) for its theoretical basis are not grounded explicitly in the literature. In this paper, we articulate appropriate accounts for these key notions and induce a conjecture so-called method adaptation referring to a process or capability in which agents holding intentions through responsive changes in, and dynamic interplays between, contexts, and method fragments develop a situated fragment for a specific project situation. As concluding remarks, theoretical implications of method adaptation are discussed.
\end{abstract}

\section{Introduction}

This research is concerned with situated method development, which is a particular subject in the research domains of information systems development [1] and method engineering [2], aiming to contribute to the understanding of how to achieve a method that fits a project situation. It has been acknowledged as a promising research endeavor to overcome a long-standing problem with information systems development (ISD) methods [2]. That is, as methods are not used as prescribed in practice, they fall short in supporting practitioners in the development of information systems for, for instance, a globally networked organisation using new development approaches such as agile systems development.

Please use the following format when citing this chapter:

Aydin, M. N., 2007, in IFIP International Federation for Information Processing, Volume 244, Situational Method Engineering: Fundamentals and Experiences, eds. Ralyté, J., Brinkkemper, S., Henderson-Sellers B., (Boston Springer), pp. 49-63. 
While new methods are promoted as a panacea for well-publicized ISD failures, old ones have been criticized that they are rigid, comprehensive and are built upon the idea that a method can be used for all projects which brings on a "one-size-fits all" issue [3]. In fact a fundamental problem still remains that methods, irrespective to their preferred features (agility, state-of-the art knowledge foundations), by nature involve certain thinking and often prescribe certain actions for ISD [4]. The subject matter at hand addresses this "one-size-fits all" issue and aims to deal with how an ISD method is developed and can be supported so that the resulting method, socalled situated method, fits a project situation. The idea behind a situated method is that any prospective method to be used for a development project is subject to certain adjustments because of the fact that the method is limited to its preferred thinking and prescribed actions for ISD which cannot fully accommodate the uniqueness of a project situation. In this regard, such adjustments are needed for the method along with a premise that the resulting method can provide a well-suited means for ISD and in turn reduce the risk of its failures. As shown in the succeeding sections, the existing studies appear to lack an appropriate theoretical ground that illuminates the underpinnings of these adjustments, which are referred as method adaptation. It is this missing ground that urges us to investigate what accounts the idea of situated method development. So, the goal of this paper is to articulate its underlying key notions at a foundation level.

The research can be considered as explorative research which employs a broader view on the subject matter by applying a stratification research model and use logical arguments to induce a conjecture so-called method adaptation. Basically, the stratification model adopted in this research has four modes of the analysis of relevant research [5]: (i) a classification system which includes generic categories of those studies having affinities with method development and situated method development in particular; (ii) a taxonomy which reflects basic dimensions for studying situated method development; (iii) a conceptual system in which we critically examine the conceptual elements of a few selected studies; and finally (iv) a theoretical system which includes a generic model along with a number key constructs and their relations. Notice that conceptual system and theoretical system analysis is within the scope of this paper.

The paper is outlined as follows. Having presented the motivation of the research in this introduction section, we sketch the overall research scope as well as relevant research in the second section. It is the third section where we start presenting the articulation of key notions, and providing basic understandings of the key notions. This eventually leads us to the next step whereby we incorporate their basic understandings into a conjecture that we call method adaptation. In light of this conjecture we furthermore briefly discuss three basics models proposed in the literature and conclude with its theoretical implications and future research.

\section{Research Background}

In a practical sense, the subject of this work is about supporting (human and nonhuman) an agent to make a method work for a project situation. This is often 
performed by a project manager or other actor responsible for the project. But usually there is more than one actor involved in this task and surely more actors have stakes in the outcome of this task - a situated method. This task is usually performed at the early stages of a project and can result in, for instance, a project plan, project proposal, or system development plan.

Among all the cited problems and issues hindering a better use of methods, it is argued by many scholars that methods by nature have their own limited views on the reality of IS development Truex et al. [6] assert that: "By adopting a single engineering concept of method all of our thinking about information systems development becomes imprisoned by this one concept. The method is not only our way of thinking about systems development; it is our way of thinking about "thinking about systems development".

Scholars in both the ISD research literature (see, for example, $[7,8]$ ) and method engineering (ME) (see, for example, [4, 9]) address this issue from their own perspectives. In fact, the reactions of scholars in method engineering to problems concerning methods are set forth along with the call for 'methodology engineering' in $[10,11]$, 'method engineering' by Brinkkemper and his colleagues $[9,11,12,13]$. Kumar and Welke [9] propose that "...we need a formal (as opposed to ad-hoc) and efficient (as opposed to time and money wasting) methodology for developing ISD methods which are situation appropriate (as opposed to universal) and complete (an opposite to partial), and at the same time rely on the accumulated experience and wisdom of the past (as opposed to built from scratch)" (p. 322).

At a high level, [14] distinguishes three research domains (the ISD research, Method Engineering, and Implementation research) that contribute to an understanding of situated method development. The ISD and ME research domains provide insights into the way or process (situated) method development takes places. The ISD and Implementation research domains help us employ the content of such a way (including characteristics and/or elements used in this process). [14] indicates that situated method development related studies adopt a number of key notions (situation, context, agency, method fragment) as basic elements for their models, but their articulation along with theoretical ground needs to be done explicitly. Because of this lack of explicitness, for instance, these basic notions have been incorporated with different interpretations in research domains. What is interesting to see in this review is that most of the studies mentioning and adopting these notions fall short in incorporating the essential attributes, as we shall discuss them later on, and often do not provide explicit definitions of the terms. In particular, the notion of agency as part of situated method development is undervalued in the prevailing models. Only Baskerville and Stage [15] emphasise the matter, but as a conceptual system their proposed model requires factual validity in an empirical setting and lacks unambiguous descriptions of certain elements (situation, context). With regard to the conceptual system review mode, the common terms in the aforementioned studies are conceptualized as sensitizing notions in their model building. We claim that the treatments of these notions are partial as they provide alternative or complementary viewpoints.

We contend that the prevailing models show alternative approaches to situated method development along with their pros and cons. For the examination of alternative approaches we propose to investigate situated method development as a 
phenomenon. The examination should be done at a fundamental level where its key underlying notions are naturally revealed and articulated. This examination will provide a beginning for the foundation of the phenomenon for which we induce a conjecture in section four.

\section{Articulations of Key Notions}

A detail literature review indicates that there are four essential notions (situation, context, agency, and method fragment) underlying situated method development. We shall now examine each notion in turn. By examining we mean to understand how the notion is treated in its corresponding research domain and thereafter incorporate its meaning into our research context. For instance, to understand how the notion of situation is treated in literature, we have identified and discussed three relevant studies in the research domain of linguistics, cognitive psychology, and sociology. For the notion of context, we have discussed relevant studies in the research domain of pragmatics and decision-making. For the notion of agency, we have examined the theory of intention in the philosophy of mind. The treatments of these notions are provided in their own discourses and at different levels of detail. It should be noted that to avoid any misunderstanding on the adopted notions we stick to their original meanings and remain clear about how relevant their meanings are to our subject. Let us start with situation.

The Notion of Situation. The term situation refers to, "the way in which something is placed in relation to its surroundings" [16] or "a set of circumstances in which one finds oneself, or location and surroundings of a place" [17]. The key words are here circumstances, surroundings, and placing them in a certain way. This placement has to do with cognitive activities (i.e., making sense of surrounding, circumstances, and relating with a cognitive scheme) and/or physical activities (performing an activity to do so). In Latin the term in situ as an adverb or adjective indicates a similar meaning stating that "in the natural or original position or appropriate place" [16]. The term has been used extensively in IS research in different ways, but its meaning is often reduced to a number of factors without articulating its essential features or their interplay in relation to human knowledge and action tied to its philosophical treatment. In this sense, we briefly discuss its use in sociology, linguistics, and cognitive science, and aim to come to its essential features (Table 1).

Perhaps the most comprehensive exposition of the term to appear so far in linguistics is in [18] titled "Situation and Attitudes" and associative studies that deal with situation semantics and propose a mathematical theory of situation. In sociology, it is the work of Suchman [19], entitled "Plans and Situated Action" which introduces "situated action". In cognitive science, especially in connection with artificial intelligence, Endsley [20] and her colleagues introduce "situational awareness" to emphasize "the knowing of what is going on". Three studies in this work are representative studies which help us find three complementary views on the notion of situation. In doing so, we have been able to reason about the underlying features of situation in connection with the idea of situated method. 
Regarding the theory of situation [18], which has been applied in various areas including design theory, linguistics, and artificial intelligence [21], it aims to incorporate intentions and circumstances of the agents in the communication process. [22] recognises the need to rethink the foundations of situation semantics and provide the following definitions: "Situations are contrasted with worlds; a world determines the answer to every issue, the truth-value of every proposition. A situation corresponds to the limited parts of reality we perceive, reason about, and live in. What goes on in these situations will determine answers to some issues, but not all. (p. 1)"

Table 1. The very notion of situation in three complementary studies

\begin{tabular}{|l|l|l|}
\hline Representative study & Associated Disciplines & $\begin{array}{l}\text { Essential features of the very notion of } \\
\text { situation }\end{array}$ \\
\hline Theory of Situation & Linguistics & Partial reality, Realism, Relations \\
\hline $\begin{array}{l}\text { Situational } \\
\text { Awareness }\end{array}$ & Cognitive Psychology & $\begin{array}{l}\text { Employment of cognitive mechanisms and } \\
\text { relevant factors for human knowing }\end{array}$ \\
\hline Situated Actions & Sociology & $\begin{array}{l}\text { Interactions, Partial plans and other } \\
\text { resources subsumed and produced }\end{array}$ \\
\hline
\end{tabular}

Regarding 'situated action', Lucy Suchman [19] introduces this term to underscore that actions take place in the context of particular, concrete, and possibly material and social, circumstances. She contrasts her account with the traditional view of human actions, specifically goal-directed behaviour as studied in cognitive science, asserting that plans are taken to be either formal structures that control a purposeful action or abstractions over its instances. Alternatively, her account as drawn from ethno-methodology contends that: plans are representations of actions and in the course of situated action, representation occurs when otherwise transparent activity becomes problematic in some way. Further she asserts that a central resource for achieving the objectivity of situations is language, which stands in a generally indexical relationship to circumstances that it presupposes, produces and describes. As a consequence of the indexicality of language, mutual intelligibility is achieved on each occasion of interaction with reference to situation particulars, rather than being discharged once and for all by a stable body of sharing.

The third representative study which introduces 'situational awareness' (SA), employs the models of human thinking proposed in cognitive science [20]. By 'situational awareness' Endsly [20] means, "...perception of the elements in the environment within a volume of time and space, the comprehension of their meaning and projection of their status in the near future". She argues that although the elements of SA vary widely in several disciplines, the nature of SA and the mechanisms used for achieving it are common (for instance, perception, comprehension and projection are proposed as three 'levels' underlying SA and blended with, but different from, the decision-making perspective that SA is aimed to facilitate decision-making). By drawing on associated empirical studies, they argue that certain elements (goals, expectations, mental models, schema, and automaticity) influence SA and are vital for the agency's internal representation of state. It should be noted that SA is concerned about the state of knowledge that has to do with the 
references to confirmed schemas and the 'yet-to-be-tested' hypothesis, rather than the process of achieving this. Many factors (e.g., task under or overload, fatigue, psychological stress) may also degrade SA, but they are, as claimed, independent constructs. It is suggested that other terms like shared situational awareness, shared understanding and distributed /shared cognition should be used for a collective version of the SA as it has an originally individual focus. It is also suggested that factors like culture, experience, personality, sex, and age as 'structural factors' are different from 'situational factors' such us mood, time pressure, stress, ambiguity, etc.

Our understanding of the term situation has some commonalities with the three representative studies. That is, situation is about:

- A limited portion of the world - partial reality - as emerging over location and time

- Characterization (confined and yet-to-be-tested hypothesis)

- Subsumed and produced partial reality for planning (concerning future- and present-directed act)

An important corollary of partial reality is that a situation as constructed by the agency is about knowing of the agency and it is in the head of an agency. This view is in line with what [23] called "radical constructivism" which is developed following Kant (1724-1804), Vico (1668-1744), and Piaget (1896-1980). This view employs the basic principles of radical constructivism, such as that knowledge is not passively received either through the senses or by way of communication; the function of cognition is adaptive, tending towards fit or viability; cognition serves the subject's organisation of the experiential world, not the discovery of an objective ontological reality.

By drawing on the principles of radical constructivism, a constituent of a situation is not a thing-in-itself, but something that the cognizing subject has constructed by making distinctions and coordination in his or her perceptual field [24]. For the purpose of an analytical examination however, we see the three constituents - context, agency and method fragment- as distinct elements ('things-inthemselves') though each of them construes and includes the other two.

The Notion of Context. In a broader sense the term context refers to a collection of relevant conditions and surrounding influences that make a project situation unique and comprehensible [25]. The complexity of context as a subject has been acknowledged by many scholars, including [21]. [26] argues that relevant discussions on this subject in philosophy evolve from its narrowest meaning about the consideration of texts in linguistics, to its broadest meaning, something to do with 'situated cognition'- that is invariably situated, as elaborated in the field of pragmatism. In particular, a traditional view of the notion of context suggests that contexts are pre-existing and stable environments that perhaps include unobservable factors that cause agencies to behave in partly unpredictable ways [26]. This view appears to be akin to what [26] calls the optimistic claims stating that for all classes of cognitive tasks and processes, there is a uniform context matrix - whatever the features or factors are granted, such that for all situations in the class, the outcome of any process in the class is determined by the values taken by the matrix in the situation. 
This is often contrasted with the contemporary view which asserts that all contextual regularities, conditions and any other relevant features, are assumed to be dynamically activated and accomplished in the situation [27]. Context has also been studied as a central notion in human decision-making. [28] illuminates the dynamics of context and the employment of reasoning for 'practical' decision-making. Practical decision-making, as discussed by [28], is reminiscent of naturalistic decision-making, an adopted orientation in this work.

Different kinds of context are introduced with a duality character [29] such as 'immediate' or 'proximate' contexts. These include features pertaining to actual surroundings in situ versus 'distal' or 'mediate' contexts which cover background knowledge, cognitive frames, or assumptions about on-going, up-coming, or even priori activities relevant in situ. Another distinction is made between so-called primary and secondary context, the extent to which influencing characteristics are stable [28]. In relation to this duality character, [26] defends a 'mixed model of inquiry', which combines rationalist reliance either on fact or principles with a consideration for appropriateness to the situation at hand. This is indeed where the pragmatics view of context stands and of which several accounts are proposed. [30], for instance, advocates this view and argues that ambiguity is inherent in contextualization, decontextualization, and recontextualization (hereafter called 'contextualizing') through which one may effectively marginalize certain agencies and their legitimate interpretations by virtue of an institutionally embedded context.

Human agency is central to contextualization. In connection with this work, of course, method fragments are also considered during this contextualization. But exclusion of the agency and method fragments is in effect when the context is framed and reframed along with the cognitive structure and processes [24]. After successive approximation, this eventually leads to an appropriate context under consideration with respect to, upon, and in which the decision is made. Accordingly, cognitive structures change through the process of adaptation by assimilation and accommodation. This is boldly marked in the radical constructivism along with the principle stating that the function of cognition is adaptive and serves the agency's framing or organizing of the experiential world, not the discovery of an objective ontological reality [23]. We employ the ideas of 'contextualizing', 'framing', 'appropriation' in relation to the very notion of context.

The Notion of Agency. Cognitive elements come into place at the outset of situated cognition when contextualizing takes place in situ where the agency is supposed to make a decision and to perform actions. But what cognitive elements are manifest in human thinking and actions? It has been argued for a long time that desire and belief are the elements that have certain direct impacts on human thinking and actions. There is no doubt that beliefs and desires are always present in the cognitive structures and process with some effects, but contemporary studies in the field of the philosophy of mind, including [31] and his associates, have questioned their direct effects in the course of actions and corresponding decision- making.

Granting that human knowing, more broadly thinking, and actions are inherent in determining situation, we turn our discussion to what cognitive elements are necessary for situated method development. In principle, human thinking is subject to the complexity of interplay between many cognitive elements such as beliefs, 
norms, motives, goals, and intentions. The accounts on each term or their combinations along with counter arguments are readily available in philosophy as a reference discipline as well as in certain applied sciences (management science, IS research, organisational science) where the prospective accounts are adopted. By drawing upon the works of [32] in the philosophy of mind and Husserl (1859-1938) and proponent scholars in the philosophy science, our aim in this section is to show that as a cognitive element, the notion of intention serves best to explain the interplay between the method fragments, the agency, and the context.

In the dictionary [16] and every day language, the term intention is synonymous for volition, purpose, and significance, and indicates "a determination to act in a certain way". Other derivations and uses of the term appear as intent, intentionality, doing with an intention, or doing something intentionally. To ground explanations concerning their differences would require a long philosophical treatise which belongs to the philosophy of mind, but the treatment of intention and intentionality in [32] and [32] is relevant to our subject. The treatment of the terms intention and intentionality should be separated as the former has been articulated in relation to action, planning and practical rationality [31], and the latter is proposed in phenomenology, a particular school of thought in the philosophy. Intention is considered a state of mind (what it is to intend to something) and a characteristic of action (having an intention to do something or doing something intentionally).

'Intentionality' derives from the Latin verb 'intendere', which means "to point" or "to aim at", and Brentano (1838-1917) accordingly characterized the intentionality of mental states and experiences as their feature of each being 'directed toward something'. Intentionality in this technical sense then subsumes the everyday notion of doing something "intentionally": an action is intentional when done with a certain "intention", i.e., a mental state of "aiming" toward a certain state of affairs.

One of the most comprehensive expositions of the term intention is in the work of Michael Bratman [31]. His treatment reveals complexity and the essence of its characteristics and functions along with two forms (future- and present-directed). [31] extensively discusses his account in relation to planning theory and agent rationality, for which we cannot condense the body of literature he employs in a few pages. The forms and kinds of intention he proposed however, are especially useful for characterizing the agency action in method adaptation.

Upon the deeper examination of the idea of intending to act, which channels a future-directed form of intention, or having an intention to act, which is presentdirected action, he contends that intentions are neither desires nor beliefs but plans, and that plans have an independent place in practical thinking. One of the central facts about intentions essential for this work is that they are conduct-controlling proattitudes and serve as inputs for further practical reasoning. According to [31], distinct from normal beliefs, both desires and intentions are pro-attitudes, which have a motivational function for an act. As distinct from desires or other weak proposition attitudes such as beliefs and goals, (considered potential influencers of action) intentions are conduct-controlling pro-attitudes. As such, intentions are parts of partial plans for action, required by an agency that must make complex plans but cannot make the plans complete. The partial plans play a central role in practical reasoning, aimed at adjusting and completing prior but partial plans, and help extend 
the influence of deliberation beyond the present moment and facilitate coordination within the agent's life and, socially, between agents.

The Notion of Method Fragment. Philosophical treatment of the term method is often done implicitly while discussing the matters about, for instance, rationality of agency, reasoning in the formation of thinking and action. In fact the definition of method holds a very strong affinity with these matters, but its elaboration is beyond the scope of this work. We therefore turn to the IS research literature to articulate the notion of method and method fragment. Recall the definition of (ISD) method: an explicit way to structure one's thinking and actions. It is the one, as we term agency that has some affinity and involvement in a project [33]. The method does not do anything itself though there are certain parts of method that perform some activities together with an agent (modelling, testing, coding, etc.). What is interesting to see is that a method structures or helps someone to structure other agencies' thinking and actions. This is done together or without the others agencies at the time (t1) which occurs before the actual execution ( $\mathrm{t} 2$ ) of the structured thinking and actions. That is where an intriguing relation with $\mathrm{t} 1$ and $\mathrm{t} 2$ begins because,

- It is very optimistic to think that the context at t2 is truly taken into account in this structuring at $\mathrm{t} 1$;

- It is too idealistic to consider that the agent who makes use of the method to achieve this structure has the same intention embedded in the methods (i.e. incongruence of the agent's perceived situation with the situation held by the method);

- It is too strong, and possibly incorrect, to surmise that the agents who hopefully hold and practice in the context at $\mathrm{t} 2$ will have the same intentions as presumed.

We argue that structuring at $\mathrm{t} 1$ and under the context $\mathrm{c} 1$, one's thinking and actions to be executed at $\mathrm{t} 2$ under the context $\mathrm{c} 2$ is a yet-to-be-tested hypothesis. Namely, neither the method to be situated nor the agent who wishes to achieve a situated method can justify or even claim the structured thinking and actions will be realized as intended and contextualized. But, if this is so, what is the rationale behind a situated method?

First and foremost, a meaning of situated method is revised in that it is not with a fine-grained description of the method that we are concerned, but instead the intentions attached to a number of key deliberative actions to be appropriate to the contexts under consideration. We also note that method as inanimate agency holds 'frozen-rational' of its producer. It is necessary to explicate how this frozen-rational with its collectives are proposed to be situated, when present. If it does not include this aspect (i.e. how it is to be situated), it fails to hold the very idea of situated method. [33] criticizes methods on this matter, and proposes a framework containing four essential elements: the 'problem situation' (similar to the term context we use), the intended problem solver (methodology user), the problem-solving process (the method), and the evaluation of the above three. The proposed framework has certain interesting features pertaining to the goal, as opposed to forcing the method user to use the method, that facilitate the designer to come to her own method. For this purpose, the designer and user are encouraged to ask a number of questions and critically examine the intention of every action needed. Some examples: What are the methodology users' value sets? What believes do they hold as being "good"? For 
example, which of the economic, political, cultural, or technical values do the methodology users consider as uppermost? In this context what values do the methodologies advocate? How congruent are these with methodology users' values?

\section{Incorporating the Four Essential Notions for Method Adaptation}

As discussed above that the four essential notions are often conceived from what we call a basic or simplistic view, they need to be extended to comprehensive and possibly richer meanings. We consider the notion of 'situation' a phenomenon with which the agency perceives, reasons about, and lives in at certain time. Three complementary views on situation -Theory of Situation, Situational Awareness, and Situated Actions, summarized in Table 1, indicate underlying features of this construct, which is essentially a composite one. By employing the theory of situation [18] we contend that situation is partial reality at best which has to do with the relations among the collectives under consideration. By employing the idea of situational awareness [20], we argue that the agency needs to use all kinds of cognitive elements and mechanisms to be aware of the position held on and reason about what we intend to do. By employing the idea of situated action [19], situated method is enacted by interactions among its collectives along partial plans.

By drawing on the conception of situation we conjecture that agency, context, and fragment are essential for situated method development. Situated method is regarded as a phenomenon because it is:

- Based on partial reality construed by the agency that forms the intention in the context at a certain time and in place,

- Enacted and re-constructed for the context in which the agencies' thinking and actions are structured and referred thereof

Table 2. An extension of four essential notions for situated method development

\begin{tabular}{|l|l|l|}
\hline $\begin{array}{l}\text { Four essential } \\
\text { notions }\end{array}$ & Basic View (Simplistic) & Extension \\
\hline Situation & $\begin{array}{l}\text { characterized by a number of } \\
\text { factors that influence or are being } \\
\text { influenced by a method fragment }\end{array}$ & $\begin{array}{l}\text { the limited parts of reality that the } \\
\text { agency perceive, reason about, } \\
\text { and live in }\end{array}$ \\
\hline Context & $\begin{array}{l}\text { Described in terms of aspects or } \\
\text { collectives in the process }\end{array}$ & $\begin{array}{l}\text { dynamic interplays among } \\
\text { collectives of work practice as } \\
\text { situated and characterized by the } \\
\text { agency }\end{array}$ \\
\hline Agency & $\begin{array}{l}\text { adheres to enactment of proposed } \\
\text { fragment in the work practice }\end{array}$ & $\begin{array}{l}\text { interplays among fragments with } \\
\text { a certain intention in and for the } \\
\text { context }\end{array}$ \\
\hline $\begin{array}{l}\text { Method } \\
\text { fragment }\end{array}$ & $\begin{array}{l}\text { description of a methodical artefact } \\
\text { or any coherent part thereof }\end{array}$ & $\begin{array}{l}\text { comes into play with the agency } \\
\text { in the context when structuring } \\
\text { one's thinking and actions }\end{array}$ \\
\hline
\end{tabular}

The following summarises our conceptions of three notions (see Table 2). 
Regarding context, Andler's [34] account gives a hint about two aspects of a context: On the one hand it is perceived, and perhaps influenced by means of the agency's own fragments (fragments already used a priori by the agent) and proposed fragments (the fragments not used a priori by the agent). On the other hand it influences the agency's fragments and proposed fragments. It has then a duality character on 'to influence' and 'being influenced', which is manifest in the process of contextualizing, de-contextualizing, and re-contextualizing. In other words, this process is about 'characterization of context for situation awareness'. This characterization includes, as referred to in [20], perception, comprehension, and projection. It is this characterization that uses a number of factors considered salient to the situation at hand. Most of these characteristics are nothing more than subjective views of the situation. By drawing on the literature of social cognition, we contend that characterization remains effective when the relations among the characteristics of the situation can be present to achieve a 'yet-to-be-tested hypothesis', sometimes represented as heuristics. As time progresses in situated method development and more insights are gained along emergent attributes of the context, relations among the characteristics are subverted and (re)formed as the meanings and their importance is characterized again.

Agency, is at the heart of situated method development where it interacts with the fragments (owned and proposed) in and for the context. The agency conducts characterization of the context in which all collectives (other agencies having one of the roles as identified, methodical artefacts as shall be elaborated below), and other constituents of the situation are considered. At any moment during this characterization the agency may need to determine what to do with the fragments owned and proposed (i.e., how to structure the agents' thinking and actions in the situation foreseen). This determination is an intentional action of the situation at hand and involves a human decision-making process. We argue that the concept of intention, along with its main functions and forms (future- and present-directed), paves the way for an account of the agency theorizing the way an agent structures his own and/or the user's thinking and action for constructing a situated method. Accepting that the situation at hand and that which is foreseen (where the actions are performed intentionally whether or not the associated intentions agree with the proposed one) are partially construed and relative to the agency, uncertainty is always inherent in situated method development and in determination of the fragments. Therefore, a body of knowledge concerning 'decision-making under uncertainty' is used to understand how decision-making is achieved in situated method development. In particular, naturalistic decision-making accounts are found to be appropriate as their particular view on decision matters fits our orientation on the subject matter.

Regarding (method) fragment, which is of course, present in situated method development and is a cognitive element that presupposes agents' future-directed intentions and is materialized in different forms (template, procedure, technique, etc.). Due to this cognitive aspect, a method fragment influences the way a designer structures her thinking and actions that affect the way the user structures and realises her thinking and actions. Various intriguing interplays occur between the agency and method fragments that will be elaborated later on, but to give an example, consider a simple case where the designer adopts the fragment without any change (i.e., the 
designer role is not effective). In this case, the fragment becomes more dominant in situated method development (i.e., it directly structures its user's thinking and actions). But that is only one direction of the influence; the other manifested as the method fragment is subject to change in the execution of the proposed fragment (i.e., the proposed fragment is enacted and modified in a context). These two aspects of fragment, similar to context, show a duality of method fragment (simply, 'to influence' and 'being influenced') which manifests the process of contextualizing, de-contextualizing, and re-contextualizing of the fragment. In other words, this process is about 'characterization of method fragment for situation awareness'.

As we have incorporated basic understandings of the key notions for situated method development, we are ready to induce the proposition about the meaning of adaptation for situated method development as well as the conjecture asserting how the underlying notions can be understood better.

Proposition: Adaptation Underpinning Situated Method Development. Adaptation is essential to situated method development because the agents in a 'perfect' sense cannot arrive at matching, adjusting, and/or transferring elements of a situated method where the context is unique and relative for each agency.

Conjecture: Method Adaptation Process (MAP). Given that three concepts (context, fragment, and the agency) emphasise the idea of modifications, changes on, and interplays among them, we conjecture that the 'Method Adaptation Process', in short 'method adaptation' or 'MAP', is a process or capability in which agents holding intentions through responsive changes in, and dynamic interplays between, contexts, and method fragments develop a situated fragment for a specific project situation.

Notice that with this conjecture, we consider a situation as a collection of three essential concepts: Agent, Context, and Fragment. The conjecture does not claim how these interplays may occur, but asserts that this interplays come into an end in the form of a situated method. In the following, we refer to three studies $[35,36,14]$ to discuss briefly how these interplays can manifest in terms of models. We shall briefly mention the corresponding interplays as incorporated in their proposed models.

The interplays between context and fragment are incorporated explicitly in the Configuration Process [35]; on the other hand, the interplays between agent and context are implicit in the model. The S3 Model of Situational Method Engineering in [36] puts a special emphasis on the explicit interplay between context and fragment and on the implicit interplays between agent and fragment. This implicit mentioning of the other interplays in [35] and [36] is not surprising because the notion of agency is not central to their articulation of the idea of situated method development.

Baskerville and Stage [15] propose a social process for situated method development along with the premise that a method should be situated at the ISD level where ISD activities are carried out. Similar to the previous two basic models, characterization of a context in terms, a number of elements are suggested though their mutual relations are not addressed in such a characterization. In relation to the Social Process for Method Fragment Adaptation in [15], the process as proposed is a 
good example of a special emphasis on the explicit interplay between agent and context and on the implicit interplays between agent and fragment.

To conclude with the examination of the conjecture in relation to the basic models, we contend that as they correspond to specific interplays, they put special emphasis on the interplays between agent, context, and fragment with different degrees and explicitness. These models can be seen as specific patterns reflecting specific orientation on the subject matter.

\section{Conclusion}

This paper is concerned with theoretical underpinnings of situational method development, which concerns about how to make a method work for a project situation. In literature, various approaches, models or alike are proposed to describe or prescribe how to achieve a situated method, which is a method that fits a project situation. Based on the review of relevant studies in ISD and method engineering research domains, we point out that there is a lack of explicit articulation of key notions underlying situated method development. Eventually, upon the deeper examination of key notions in various disciplines, including cognitive psychology, philosophy of mind, and linguistics, we induce a proposition about the meaning of adaptation for situated method development and a conjecture called method adaptation. This conjecture states that situation, as a combining construct, embraces the other notions context, fragment and agency. As such, method adaptation asserts that there are intriguing interplays among these key notions. We briefly discuss the specific interplays that are found in basic models proposed for situated method development in literature. One implication of method adaptation is that method, context and the agent are not passive elements in these interplays but purposively intervene in the agent's knowledge about how to handle construction of situated method. This implies that we should advance in our thinking about the effect of method in these interplays rather than reducing its meaning to certain aspects and attributes. To show how to advance in thinking, we suggest looking beyond its 'frozen' rationale captured and often implicit in the presence of the method, and possibly capture its creator's way of structuring the intended user's thinking and actions.

This conjecture may suggest two basic directions for future research. First, it seems that this conjecture gives a hint about a need for a generic model that explains possible interplays among the key notions [37]. If this is possible, the existing basic models can be considered as specific patterns that can be induced from a generic model [38]. Description of a generic model should be made in an ambiguous way so that the comparison of the generic model with other models can be made explicitly. Second, empirical justifications of the specific interplays may be needed to show the feasibility of studying certain models in practice. In fact, this is one of the immediate needs for conducting relevant research to better understand how situated method development occurs in practice. The deeper articulation of its underlying key notions may accommodate the one intending to carry out follow-up research. 


\section{References}

1. D. Avison, D and G. Fitzgerald, Reflections on Information Systems Development 19882002, in: Information Systems Development - Advances in Methodologies, Components, and Management, edited by M. Kirikova, J. Grundspenkis, W. Wojtkowskiet (Kluwer Academic/ Plenium Publishers, 2002), pp. 1-11.

2. K. Kumar and R. J. Welke, Methodology Engineering: A Proposal for Situation-Specific Methodology Construction, in: Challanges and Strategies for Research in Systems Development Method, edited by W. W. Cotterman, J. A. Senn (John Wiley \& Sons, 1992).

3. G. J. Hidding, Reinventing Methodology: Communications of the ACM, 40(11) (1997)

4. B. Fitzgerald, The Use of Systems Development Methodologies in Practice: A Field Study. Information Systems Journal, 7, 201-212 (1997).

5. J. Webster and R. T. Watson, Analyzing the Past to Prepare for the Future: writing a Literature Review, MIS Quarterly 26(2), xiii-xxiii (2002).

6. D. Truex, R. Baskerville, and J. Travis, Amethodical system development: the deffered meaning of systems development method. Accounting, Management \& Technology, 10,5379 (2000).

7. J. Iivari and H. Linger, Knowledge Work as Collaborative Work: A Situated Activity Theory View. HICCS99, Hawaii, USA (1999).

8. T. W. Olle, H. G., Sol, and A. A. Verrijn-Stuart, Information Systems Design Methdologies: A Comparative Review. Amsterdam, North-Holland (1982).

9. S. Brinkkemper, Method Engineering: Engineering of Information Systems Development Methods and Tools, Journal of Systems and Software, 38, 275-280 (1996).

10. R. J. Welke, K. Kumar and H. van Dissel, Methodology Engineering: Een voorstel om te komen tot situationeel specifieke methode-ontwikkeling, Informatie, 33(5), 11-20 (1981).

11. K. Kumar and R. J. Welke, Methodology Engineering: A Proposal for Situation-Specific Methodology Construction. in: Challanges and Strategies for Research in Systems Development Method, edited by W. W. Cotterman, J. A. Senn (John Wiley \& Sons, 1992).

12. C. van Slooten, S. Brinkkemper, A Method Engineering Approach to Information Systems Development, in: Information System Development Process, by N. Prakash, C. Rolland and B. Pernici (Elsevier Science Publishers, North-Holland, 1993).

13. F. Harmsen, S. Brinkkemper, and H. Oei, Situational Method Engineering for Information Systems Projects. in: Methods and Associated Tools for Information Systems Life Cycle, edited by T. W. Olle and A. V. Stuart (North-Holland, Amsterdam, 1994), pp. 169-194.

14. M. N. Aydin, F. Harmsen, and J. van Hillegersberg, Taxonomic Dimensions for Studying Situational Information Systems Development, In: Situational Method Engineering: Fundamentals and Experiences, edited by J. Ralyté, S. Brinkkemper and B. HendersonSellers, IFIP Series in print (2007).

15. R. Baskerville and J. Stage, Accommodating emergent work practices: Ethnographic choice of method fragments. In: Realigning research and practice: The social and organisational perspectives (Kluwer Academic Publishers, Boston, 2001), pp. 11-27.

16. Merriam-Webster, (February 13, 2005); http://www.m-w.com.

17. OED - Oxford English Dictionary, (Feb 13, 2005; http://www.oed.com

18. J. Barwise and J. Perry, Situations and Attitudes (Cambridge, MIT-Bradford, 1983).

19. L. A. Suchman, Plans and situated actions: The problem of human-machine communications (Cambridge University Press, Cambridge, 1987).

20. M. R. Endsley, Design and Evaluation for Situation Awareness Enhancement. the Proceedings of the Human Factors Society 32nd Annual Meeting, Human Factors Society, Santa Monica, CA, 97-101 (1988).

21. P. R. Cohen and H. J. Levesque, Persistence, Intention and Commitment. In: Proceedings of Timberline workshop on Reasoning about Plans and Actions, 297-338 (1987). 
22. J. Perry, Semantics and Situation, Routledge Encyclopedia of Philosophy, retrieved from http://www-csli.stanford.edu/ john/PHILPAPERS/sitsem.pdf on March 13, 2002, (1987)

23. E. von Glasersfeld, Piaget's Legacy: Cognition as Adaptive Activity In: A. Riegler, M. Peschl and A. von Stein (Eds.). Understanding representation in the cognitive sciences Does representation need reality? New York/Dordrecht: (Kluwer Academic/Plenum Publishers, 1997) 283-287.

24. J. Piaget, Piaget's Theory. In P. Mussen (Ed.) Handbook of child psychology. (Wiley, 1983).

25. L. Hasher and R. T. Zacks, Automatic Processing of Fundamental Information: the Case of Frequency of Occurrence. American Psychologist, 39(12), 1372-1388, (1984).

26. B. Rogoff and J. Lave, Everyday Cognition: Its Development in Social Context (Harvard University Press, 1984).

27. P. Linell and D. P. Thunqvist, Moving in and Out of Framings: Activity Contexts in Talks with Young Unemployed People Within a Training project. Journal of Pragmatics, 35(3), 409-434 (2003).

28. J. -Ch. Pomerol and P. Brézillon, About some relationships between knowledge and context. Modeling and Using Context (CONTEXT-01). Lecture Notes in Computer Science, Springer Verlag, 461-464 (2001).

20. E. Schegloff, In another context, Duranti, in: Rethinking Context: Language as an Interactive Phenomenon, edited by A. Goodwin, (Cambridge: Cambridge University Press, 1992), pp. 193-227

30. J. L. Mey, Context and (dis)ambiguity: a pragmatic view, Journal of Pragmatics, 35, 331347.

31. M. Bratman, Intention, Plans and Practical Reason. Harvard University Press (1987).

32. Morrison, James C. (1970) Husserl and Brentano on Intentionality. Philosophy and Phenomenological Research, 31, 27-46 (2003).

33. N. Jayaratna, Understanding and Evaluating Methodologies (McGraw-Hill, Berkshire 1994).

34. D. Andler. Context: the case for a principles epistemic particularism, Journal of Pragmatics, 35(3), 349-371 (2003)

35. C. van Slooten, Situated Methods For Systems Development, Doctoral Dissertation, University of Twente (1995).

36. F. Harmsen, Situational Method Engineering. (Moret Ernst \& Young Management Consultants, Utrecht ,1997).

37. Aydin, M. N. Decision-Making and Support for Method Adaptation, PhD Dissertation, University of Twente, ISBN: 90-365-2375-3 (2006)

38. Mirbel and J. Ralyté, Situational Method Engineering: Combining assembly-based and roadmap driven approaches, Requirements Engineering, 11(1):58-78 (2006). 